\title{
INSULATING CIRCULAR RUGS
}

\author{
G. J. WEIR'
}

(Received 11 August 1982; revised 18 August 1982)

\begin{abstract}
A thin, partially insulating circular rug is placed on a uniform half space up through which a steady heat flow passes. The corresponding dual integral equations are solved using Tranter's method, finite Legendre transforms and Mellin-Barnes contour integrals. An untabulated Bessel (or Stieltjes) transform similar to the discontinuous WeberSchafheitlin integral is evaluated, and a simple expression derived for the rug's surface temperature.
\end{abstract}

\section{Introduction}

The aim of this paper is to derive the perturbation to temperature due to a thin, circular, partially insulating rug lying on a uniformly conducting floor. This problem originated from building research, but it may also relate to some problems associated with terrestrial heat flow. The mixed boundary conditions lead to dual integral equations which are solved by Tranter's method, complex variable theory and finite Legendre transforms.

In Section 2 we present the simplifying assumptions and associated equations; in Section 3 we evaluate a Bessel (or Stieltjes) transform and derive a simple expression for surface temperature values, while in Section 4 we summarise our results and present some numerical examples.

\footnotetext{
'Applied Mathematics Division, Department of Scientific and Industrial Research, P.O. Box 1335, Wellington, New Zealand.

(C) Copyright Australian Mathematical Society 1983
} 


\section{The dual integral equations}

A small, thin, circular, partially insulating rug of radius $R_{0}$ lies on a thick uniformly conducting floor up through which a uniform heat flux $Q$ originates at depth. We are primarily interested in the surface temperature rise under the rug if radiation boundary conditions are applied at the upper surface.

In a cylindrical coordinate system $(Z, R, \phi)$, the equations arc:

steady-state conduction,

$$
\nabla^{2} T=0, \quad Z<0,
$$

uniform upper temperature,

$$
T=T_{0}, \quad Z>0,
$$

steady lower heat influx,

$$
-K T,_{z}=Q, \quad Z \rightarrow-\infty,
$$

radiation boundary conditions,

$$
\begin{array}{ll}
K T,_{Z}=-h_{0}\left(T-T_{0}\right), & Z=0,0<R<R_{0}, \\
K T,{ }_{z}=-h_{1}\left(T-T_{0}\right), & Z=0, R>R_{0},
\end{array}
$$

where $T$ is temperature, $K$ thermal conductivity and $h_{0}, h_{1}$ are respectively the linear heat transfer coefficients for the rug and floor. These equations are non-dimensionalised through the definitions

$$
\begin{gathered}
T=-Q Z / K+T_{0}+Q / h_{1}+Q R_{0}\left(1-h_{0} / h_{1}\right) \chi / K, \\
(Z, R)=R_{0}(z, r), \\
\alpha=h_{0} R_{0} / K, \\
\beta=h_{1} R_{0} / K,
\end{gathered}
$$

to give

$$
\begin{aligned}
\nabla^{2} \chi & =0, \quad z<0, \\
\chi,,_{z} & =0, \quad z \rightarrow-\infty, \\
\chi,,_{z} & =-\alpha \chi+1, \quad z=0,0<r<1, \\
\chi,,_{z} & =-\beta \chi, \quad z=0, r>1,
\end{aligned}
$$

where we have ignored the trivial cases $h_{0}=h_{1}$ or $R_{0}=0$.

Since the problem is described by the two non-dimensional parameters $\alpha, \beta$ there are three length scales: $R_{0}, K / h_{0}$ and $K / h_{1}$. We have assumed that the vertical length scale is much greater than these three lengths. The two limiting cases occur when $R_{0}$ is either much less $(\alpha, \beta \ll 1)$ or much greater $(\alpha, \beta \gg 1)$ than the radiative length scales $K / h_{0}$ or $K / h_{1}$. 
When $\alpha, \beta \ll 1$ then $\chi$ is approximately independent of $\alpha$ and $\beta$, and Bessel transforms yield for the leading term

$$
\chi(r, z)=\int_{0}^{\infty} e^{p z} J_{0}(r p) J_{1}(p) d p / p
$$

and so from Oberhettinger [2],

$$
\begin{aligned}
& \chi(r, 0)=(2 / \pi) \mathbf{E}(r), \quad 0<r<1, \\
\chi(r, 0)= & (2 r / \pi)\left(\mathbf{E}\left(r^{-1}\right)-\left(1-r^{-2}\right) \mathbf{K}\left(r^{-1}\right)\right), \quad r>1, \\
\chi & \chi(0, z)=1+z / \sqrt{1+z^{2}}, \quad z<0,
\end{aligned}
$$

where $\mathbf{E}$ and $\mathbf{K}$ are the complete elliptic integrals.

The author has been unable to proceed simply when $\alpha, \beta \gg 1$, although the boundary conditions (equations (7) and (8)) then suggest that $\chi(0, r<1) \simeq \alpha^{-1}$, and $\chi(0, r>1) \simeq 0$. This will be confirmed below.

To proceed we shall follow Tranter [3] and represent $\chi$ by the Bessel transform

$$
\chi=\int_{0}^{\infty} f(p) e^{p z} J_{0}(r p) d p /(p+\beta),
$$

whence equations (7) and (8) give the dual integral equations

$$
\int_{0}^{\infty}(p+\alpha) f(p) J_{0}(r p) d p /(p+\beta)=1, \quad 0<r<1,
$$

and

$$
\int_{0}^{\infty} f(p) J_{0}(r p) d p=0, \quad r>1 .
$$

When $\alpha=\beta$, equations (10)-(12) are satisfied by $f(p)=J_{1}(p)$, and Tranter's method suggests setting

$$
f(p)=\sum_{m=0}^{\infty} a_{m} J_{2 m+1}(p),
$$

which automatically satisfies equation (12) and so we have to solve

$$
\sum_{m=0}^{\infty} a_{m} \int_{0}^{\infty}(p+\alpha) J_{2 m+1}(p) J_{0}(r p) d p /(p+\beta)=1 .
$$

Finally, the finite Legendre transforms

$$
\int_{0}^{1} r P_{n}\left(1-2 r^{2}\right) d r=\frac{1}{4} \int_{-1}^{1} P_{n}(x) P_{0}(x) d x=\frac{1}{2} \delta_{n, 0},
$$

and

$$
\int_{0}^{1} r J_{0}(r p) P_{n}\left(1-2 r^{2}\right) d r=p^{-1} J_{2 n+1}(p),
$$


where $P_{n}$ are Legendre functions of order $n$ and $\delta_{n, 0}$ is the Kronecker delta function, together with the discontinuous Weber-Schafheitlin integral

$$
\int_{0}^{\infty} J_{2 m+1}(p) J_{2 n+1}(p) d p / p=\delta_{n, m} /(2+4 n),
$$

then reduce equation (14) to the algebraic system

$$
\alpha a_{n}+(2+4 n)(\beta-\alpha) \sum_{m=0}^{\infty} a_{m} J_{m n}(\beta)=(1+2 n) \beta \delta_{n .0},
$$

where

$$
J_{m n}(\beta)=\int_{0}^{\infty} J_{2 m+1}(p) J_{2 n+1}(p) d p /(p+\beta) .
$$

\section{Evaluation of $J_{m n}(\beta)$}

The Stieltjes transform $J_{m n}(\beta)$ in equation (19) is an obvious generalisation of the Bessel transform in equation (17), and can be evaluated by using the Mellin-Barnes contour integral representation (Watson [4], Wooding [5]) for the product of Bessel functions with the same argument. We find

$$
\begin{aligned}
& J_{m n}(\beta)=\frac{1}{2 \pi i} \\
& \quad \times \int_{0}^{\infty} \int_{c-i \infty}^{c+\infty} \frac{\Gamma(-s) \Gamma(2 m+2 n+2 s+3)(p / 2)^{2 m+2 n+2 s+2} d s d p}{\Gamma(2 m+s+2) \Gamma(2 n+s+2) \Gamma(2 m+2 n+s+3)(p+\beta)},
\end{aligned}
$$

and requiring that

$$
-m-n-\frac{3}{2}<\operatorname{Re}(s)<-m-n-1
$$

guarantees that the integral with respect to $p$ is absolutely convergent, as well as that the poles of $\Gamma(-s)$ lie to the right of the contour while the poles of $\Gamma(2 m+2 n+2 s+3)$ lie to the left. The order of integration in equation (20) can then be reversed and, since $-1<\operatorname{Re}(2 m+2 n+2 s+2)<0$,

$$
\int_{0}^{\infty} p^{2 m+2 n+2 s+2} d p /(p+\beta)=-\pi \beta^{2 m+2 n+2 s+2} \operatorname{cosec}(2 \pi s),
$$

and so

$J_{m n}(\beta)$

$$
\begin{aligned}
= & \frac{\pi}{2 i} \int_{c-i \infty}^{c+\infty} \frac{\Gamma(2 m+2 n+2 s+3)}{\Gamma(s+1) \Gamma(2 m+s+2) \Gamma(2 n+s+2) \Gamma(2 m+2 n+s+3)} \\
& \times \frac{(\beta / 2)^{2 m+2 n+2 s+2} d s}{\sin 2 \pi s \sin \pi s} .
\end{aligned}
$$


For $l=0,1, \ldots, \sin \pi s$ contributes poles at $s=-m-n-1+l ; \sin 2 \pi s$ contributes poles at $s=-m-n-1-l / 2 ; \Gamma(s+1)$ contributes $m+n$ zeros at $s=-m-n-1, \ldots,-1 ;$ and when $m \neq n$ either $\Gamma(2 m+s+2)$ or $\Gamma(2 n+s+2)$ contributes $|m-n|$ zeros at $s=-m-n-1, \ldots,-2-2 \min \{m, n\}$. Thus there are single poles for $s=-m-n-\frac{1}{2}+l$, double poles for $s=l$ and $1+$ $2 \min \{m, n\}$ single poles for $s=-1-2 \min \{m, n\}, \ldots,-1$. The contour in equation (21) is deformed to the right to surround the appropriate poles in a clockwise manner, and since the contribution on a large semi-circle to the right tends to zero,

$$
J_{m n}(\beta)=S+Z D+D,
$$

where $S$ denotes (contributions from) single poles, $Z D$ the $1+2 \min \{m, n\}$ single poles and $D$ double poles.

We find

$$
\begin{gathered}
S=\frac{\pi}{2} \sum_{l=0}^{\infty} \frac{(-1)^{l+m+n+1} \Gamma(2 l+2)}{\Gamma(l+m+n+5 / 2) \Gamma(l+m-n+3 / 2)} \\
\quad \times \frac{(\beta / 2)^{2 l+1}}{\Gamma(l-m+n+3 / 2) \Gamma(l-m-n+1 / 2)}, \\
Z D=\frac{1}{2} \sum_{l=0}^{2 n} \frac{\Gamma(2 l+2 m-2 n+1) \Gamma(2 n-l+1)(\beta / 2)^{2 l+2 m-2 n}}{\Gamma(l+2 m+2) \Gamma(l+2 m-2 n+1) \Gamma(l+1)}, \\
F^{\prime}(l) / F(l)=\frac{1}{2} \sum_{l=0}^{\infty}(-1)^{l+1} F^{\prime}(l), \\
\quad-\psi \log (\beta / 2)+2 \psi(2 m+2 n+2 l+3)-\psi(l+1) \\
F(l)=\frac{\Gamma(2 m+2 n+2 l+3)(\beta / 2)^{2 m+2 n+2 l+2}}{\Gamma(l+1) \Gamma(2 m+l+2) \Gamma(2 n+l+2) \Gamma(2 m+2 n+l+3)},
\end{gathered}
$$

where $\psi$ is the logarithmic derivative of the gamma function and in equation (24) we have assumed for notational convenience that $m \geqslant n$. Equations (22)-(27) reduce to equation (17) when $\beta=0$, and equation (19) has been numerically integrated in a number of instances to check equations (22)-(27). Consequently, the coefficients $a_{n}$ in equation (18) can now be found.

From equations (10) and (13),

$$
\chi \equiv \sum_{m=0}^{\infty} a_{m} \chi_{m}=\sum_{m=0}^{\infty} a_{m} \int_{0}^{\infty} J_{2 m+1}(p) J_{0}(r p) e^{p z} d p /(p+\dot{\beta})
$$


For $z<0, \chi_{m}$ can be evaluated by expanding the product of Bessel functions in a power series in ( $p / 2)$ (Watson [4], page 148(2)) to give (Gradshteyn and Ryzhik [1], page 312,5) $\chi_{m}$ as an infinite series of exponential integrals-essentially an asymptotic expansion in $z$. The author has not done this, however, and considered only surface values of $\chi$.

To this end, define the coefficients $b_{m n}$ through the equation

$$
\begin{aligned}
\chi_{m}(r, 0) & =\int_{0}^{\infty} J_{2 m+1}(p) J_{0}\left(r_{p}\right) d p /(p+\beta) \\
& =\sum_{n=0}^{\infty} b_{m n} P_{n}\left(1-2 r^{2}\right), \quad 0<r<1,
\end{aligned}
$$

and taking the finite Legendre transform of equation (29) gives

$$
\beta b_{m n}=\delta_{m, n}-(4 n+2) J_{m n}(\beta),
$$

where equations (16), (17), (19) and the orthogonality of Legendre functions on $[-1,1]$ have been used. Finally, from equations (28), (29), (30) and (18) we obtain the simple result

$$
1+(\beta-\alpha) \chi(r, 0)=\sum_{m=0}^{\infty} a_{m} P_{m}\left(1-2 r^{2}\right), \quad 0<r<1 .
$$

\section{Discussion}

For values of $\alpha, \beta$ of order unity or less, the expressions in Section 3 are appropriate, but when $\alpha, \beta$ was much greater than unity we used equation (19) for numerical calculations.

From equation (31), the rug's surface temperature follows directly from the coefficients $a_{m}$ which are calculated from equation (18) and (19). In Table 1 we set $\beta=2 \alpha$, and it can be seen that the coefficients $a_{m}$ are

TABLE 1

Coefficients $a_{m}$ when $\beta=2 \alpha$

\begin{tabular}{|c|c|c|c|c|c|}
\hline $\begin{array}{c}\beta \\
m\end{array}$ & 0.01 & 0.1 & 1 & 10 & 100 \\
\hline 0 & 1.004 & 1.047 & 1.236 & 1.691 & 1.936 \\
\hline 1 & $8.5 \times 10^{-4}$ & $8.3 \times 10^{-3}$ & $6.6 \times 10^{-2}$ & $1.8 \times 10^{-1}$ & $8.6 \times 10^{-2}$ \\
\hline 2 & $-1.2 \times 10^{-4}$ & $-1.2 \times 10^{-3}$ & $-1.3 \times 10^{-2}$ & $-8.1 \times 10^{-2}$ & $-8.4 \times 10^{-2}$ \\
\hline 3 & $4.1 \times 10^{-5}$ & $4.1 \times 10^{-4}$ & $4.5 \times 10^{-3}$ & $3.9 \times 10^{-2}$ & $7.1 \times 10^{-2}$ \\
\hline 4 & $-1.9 \times 10^{-5}$ & $-1.9 \times 10^{-4}$ & $-2.1 \times 10^{-3}$ & $-2.0 \times 10^{-2}$ & $-6 \times 10^{-2}$ \\
\hline 5 & $1.0 \times 10^{-5}$ & $1.0 \times 10^{-4}$ & $1.1 \times 10^{-3}$ & $1.2 \times 10^{-2}$ & $5 \times 10^{-2}$ \\
\hline
\end{tabular}


rapidly decreasing functions of $m$. Also, from Table 1 , when $\alpha, \beta$ are much smaller than unity then to first order $a_{m} \simeq \delta_{m, 0}$ and when $\alpha, \beta$ are much greater than unity then to first order $a_{m} \simeq(\beta / \alpha) \delta_{m, 0}$; both of which agree with equation (31) and the discussion on limiting cases in Section 2.

Finally, if the rug has thermal conductivity $K_{R}$ and a small non-zero thickness $\Delta Z$ then an approximation to the temperature follows by replacing equation (1) by

$$
T=-Q Z / K+T_{0}+Q / h_{1}+Q R_{0}\left(1-h_{0} / h_{1}+h_{0} \Delta z / K_{R}\right) \chi / K,
$$

and retaining all other numbered equations unchanged.

\section{References}

[1] I. S. Gradshteyn and I. M. Ryzhik, Tables of integrals, series and products (Academic, New York, 1980).

[2] F. Oberhettinger, Tables of Bessel transforms (Springer, Berlin, 1972).

[3] C. J. Tranter, Integral transforms in mathemattcal physics (Methuen, London; John Wiley, New York, 1962).

[4] G. N. Watson, A treatise on the theory of Bessel functions (Cambridge University Press, Cambridge, 1944).

[5] R. A. Wooding, "Steady infiltration from a shallow circular pond," Water Resources Res. 4 (1968), 1259-1273. 